\title{
EXPORTAÇÃO DE NUTRIENTES FOLIARES EM DIFERENTES TIPOS DE PODA NA CULTURA DA ERVA-MATE ${ }^{1}$
}

\author{
NUTRIENT EXPORTATION IN DIFFERENT PRUNNING SYSTEMS OF IleX \\ paraguariensis TREES
}

\author{
Jorge Luiz Moretti de SOUZA ${ }^{*}$ \\ Marla Alessandra de $\mathrm{ARAUJO}^{3}$ \\ Gilvano Ebling BRONDANI ${ }^{4}$ \\ Carlos Bruno REISSMANN ${ }^{5}$ \\ Agenor MACCARI JÚNIOR ${ }^{6}$ \\ Clauriane Stele WOLF $^{7}$
}

\begin{abstract}
RESUMO
O tipo de poda na cultura de erva-mate apresenta forte influência na exportação de nutrientes. No entanto, pouco se conhece a respeito dessas características, bem como da adubação de reposição em determinados tipos de manejos. $O$ trabalho teve como objetivos: (i) quantificar a exportação de nutrientes ( $\mathrm{N}, \mathrm{P}, \mathrm{K}, \mathrm{Ca}$ e $\mathrm{Mg}$ ) em plantas de erva-mate quando submetidas a diferentes sistemas de podas, e (ii) comparar os valores exportados de N, P e K com a adubação recomendada para a espécie a partir da análise do solo. O experimento foi instalado em Guarapuava-PR em um erval de 15 anos de idade. Para tanto, utilizou-se o delineamento blocos ao acaso, com três blocos contendo 12 plantas amostradas por parcela. Os tratamentos testados consistiram de cinco tipos de poda: $\mathrm{T}_{1}$ - Toalete, $\mathrm{T}_{2}$ - Poda $70 \%, \mathrm{~T}_{3}$ - Recepa, $\mathrm{T}_{4}$ - Poda $90 \%$ e $\mathrm{T}_{5}$ - Schier. Como principal resultado pôde-se observar que a poda Recepa exportou cerca de $70 \%, 9,9 \%, 45,8 \%$ e $31,6 \%$ a mais de biomassa e nutrientes (N, P, K, Ca e Mg) do que as podas Toalete, Poda 70\%, Desfolha $90 \%$ e Schier, respectivamente. Em relação à adubação recomendada a partir da análise do solo e teores de nutrientes exportados pelas folhas, constatou-se coerência para os nutrientes N (Poda Schier) e K (Poda 70\% e Recepa). Contudo, os valores de P apresentaram incoerência, independente do tipo de poda testada. Na Poda 70\%, a mais utilizada na região de Guarapuava-PR, constatou-se déficit de $19,0 \%$ para o nutriente $\mathrm{N}$.
\end{abstract}

Palavras-chave: posição da copa; biomassa; teores; adubação.

\section{ABSTRACT}

The pruning system of the llex paraguariensis trees shows strong influence on the nutrients uptake. However, there is little information about these as well as about the replacement fertilization in some management systems. The objectives of this work were: (i) to quantify the nutrients uptake $(\mathrm{N}, \mathrm{P}, \mathrm{K}, \mathrm{Ca}$ and $\mathrm{Mg}$ ) for llex paraguariensis trees in different pruning systems, and (ii) to compare the N, P and K uptake values with the recommended by fertilization based on the soil analysis. The experiment was installed in Guarapuava-PR, in a 15 years old stand, with three blocks, being 12 plants sampled per plot. The treatments were five pruning systems: T1 - Cleaning Pruning, T2 - 70\% Pruning, T3 - Reduction Pruning, T4 - $90 \%$ Prunning and T5 - "Schier". The Reduction pruning treatment exported approximately $70 \%, 9.9 \%, 45.8 \%$ and $31.6 \%$ more biomass and nutrients (N, P, K, Ca and Mg) than Cleaning Pruning, 70\% Pruning, 90\% Pruning and "Schier" Pruning treatments, respectively. Related to the amount of nutrients recommended by fertilization based on soil analysis and the amount of nutrients exported by the leaves, it was observed similar results for N ("Schier" pruning) and K (70\% Pruning and Reduction). However, the $P$ values were not similar, regardless of the pruning system tested. In the $70 \%$ Pruning system, the most used in the region of Guarapuava-PR, was observed a deficit of $19.0 \%$ for $\mathrm{N}$.

Key-words: canopy position; biomass; macronutrients; fertilization.

\footnotetext{
Trabalho desenvolvido na Universidade Federal do Paraná - UFPR, Curitiba, Paraná

${ }^{2}$ Engenheiro Agrícola, Doutor em Agronomia, Professor do Departamento de Solos e Engenharia Agrícola, Setor de Ciências Agrárias, Universidade Federal do Paraná (DSEA/SCA/UFPR). Rua dos Funcionários, 1540. Cep 80035-050, Curitiba, PR, Brasil. e-mail: jmoretti@ufpr.br *Autor para correspondência.

${ }^{3}$ Engenheira Agrônoma, Mestranda do Programa de Pós-Graduação em Ciências do Solo, Universidade Federal do Paraná, UFPR, Bolsista CNPq. E-mail: marla.agro@bol.com.br.

${ }^{4}$ Engenheiro Florestal, Mestrando do Programa de Pós-Graduação em Engenharia Florestal, Universidade Federal do Paraná, UFPR, Bolsista CNPq. E-mail: gebrondani@yahoo.com.br.

${ }^{5}$ Engenheiro Florestal, Doutor em Nutrição Florestal, Professor Sênior do DSEA / SCA / UFPR.

${ }^{6}$ Engenheiro Agrônomo, Doutor em Tecnologia Pós-Colheita, Professor do DSEA / SCA / UFPR.

${ }^{7}$ Engenheira Agrônoma, Aluna do curso de Pós-Graduação em Ciência do Solo da UFPR.
} 


\section{INTRODUÇÃO}

Espécie nativa brasileira e pertencente à família Aquifoliaceae, a erva-mate (Ilex paraguariensis St. Hil.) representa grande importância econômica, social e ecológica em toda a região sul do Brasil. Particularmente no Estado do Paraná, essa cultura apresenta papel significativo no setor agrícola, sendo considerada uma atividade importante para pequenos produtores, uma vez que utiliza a mão-de-obra familiar no campo, além de gerar mais de 700 mil empregos na indústria (DONADUZZI et al., 2003; PASINATO, 2003).

No entanto, o sistema de cultivo dos ervais nativos entrou em decadência e, somente após o aparecimento de novas tendências de mercado e consumo, a demanda aumentou e permitiu o ressurgimento da atividade ervateira, estimulando o seu plantio com emprego de práticas de adensamento, florestamento e reflorestamento de áreas de toda a Região Sul do Brasil.

No Paraná existem, aproximadamente, 176 municípios que cultivam ervas, distribuídos em 11 núcleos regionais de administração: Campo Mourão, Cascavel, Curitiba, Francisco Beltrão, Guarapuava, Irati, Ivaiporã, Pato Branco, Ponta Grossa, Toledo e União da Vitória (PARANÁ, 1997). Dentre esses núcleos, a cultura da erva-mate apresenta elevada importância sócio-econômica na região de Guarapuava, destacando-se como uma das principais fontes de renda dos agricultores e trabalhadores rurais (RODIGHERI et al., 1995). Contudo, mesmo considerando as mudanças ocorridas no sistema de produção da erva-mate, tanto no Estado do Paraná como na região de Guarapuava, ainda ocorrem práticas inadequadas no seu manejo, resultando em fatores negativos no avanço da silvicultura da espécie.

Sob esse enfoque, as modalidades de poda, por exemplo, estão intimamente relacionadas às fases de crescimento da planta e podem ser realizadas desde a fase de planta jovem até sua senescência. Dentre as diferentes modalidades de poda destacam-se as podas de formação em viveiro, formação a campo, de exploração e podas de rejuvenescimento (DA CROCE e FLOSS, 1993).

A poda de produção ou formação (Poda $70 \%$ ) é a mais utilizada e está mais próxima da realidade dos produtores da região de Guarapuava. No entanto, ainda faltam informações mais detalhadas e precisas que possam indicar ao produtor a melhor forma de manejar o erval. Por isso, devido a uma série de dúvidas e hábitos culturais, a poda de produção ou formação é realizada, geralmente, por agricultores e técnicos empregandose uma série de variações, tais como a poda tradicional, em nível e limpeza, objetivando maior produção e redução de custos (DA CROCE e FLOSS, 1999).

Estudos visando avaliação nutricional de plantas de erva-mate são importantes, visto que, além da prática da poda resultar em grande extração de material foliar, também é responsável pela exportação de muitos nutrientes. Esse fato justifica uma investigação por meio da análise de nutrientes para verificar a necessidade de adubação, a fim de manter a capacidade produtiva da planta (REISSMANN et al.,1985; CAMPOS, 1991; GOMES et al., 1997; BISSO e SALET, 2000).

$O$ fato de a erva-mate ser uma cultura que tem suas folhas e ramos finos retirados regularmente, implica a exportação maciça de nutrientes, ficando a reposição nutricional necessária para manter a produtividade (GAIAD e LOPES, 1986). CARPANEZZI (1997) salienta a existência de lacunas técnicas na cultura de ervamate e acrescenta que existem muitas dúvidas quanto à condução do manejo arquitetônico das plantas e adubações de reposição. Segundo o autor, estes fatos indicam a necessidade de maior desenvolvimento de pesquisas enfocando principalmente assuntos relacionados à sustentabilidade ambiental do sistema ervateiro e a rentabilidade do produtor.

A análise de solo é o procedimento que permite determinar em que nível se encontram os teores dos nutrientes no solo (VETTORI, 1969; TOMÉ JÚNIOR, 1997; COMISSÃO DE QUÍMICA E FERTILIDADE DO SOLO - RS/SC, 2004). As informações advindas das análises de solo, quando conjugadas à análise foliar, auxiliam o monitoramento do sistema de produção agrícola e na avaliação de alterações ocorridas nos teores dos nutrientes presentes no solo (MARQUES e BENGHI, 2003). Desta forma, pode-se manter um programa de adubação que permita uma produção sustentável e, conseqüentemente, gerar maior lucratividade.

A exploração ecológica e economicamente sustentável da erva-mate é possível e poderá viabilizar o aumento da renda familiar dos agricultores, mediante menores custos e maior eficiência produtiva. Assim, a planta de erva-mate poderá fornecer produtos sem perder sua capacidade produtiva, representando uma alternativa de renda estável, com longo período de exploração (LOURENÇO et al., 1997).

$O$ objetivo do presente trabalho consistiu em: (i) quantificar a exportação de nutrientes $(\mathrm{N}, \mathrm{P}$, $\mathrm{K}$, Ca e Mg) em plantios de erva-mate (Ilex paraguariensis St. Hil.) submetidos a diferentes sistemas de poda; e (ii) relacionar os valores exportados de N, P e K com a adubação recomendada a partir da análise do solo.

\section{MATERIAL E MÉTODOS}

O experimento foi instalado no ano de 2002 e conduzido na Fazenda São José, situada na Serra da Esperança, município de Guarapuava-PR, a 25ํㅜㅇ 23' 36" de latitude sul e 51 27' 19" de longitude oeste, $1120 \mathrm{~m}$ de altitude e $286 \mathrm{~km}$ de Curitiba. O clima da região é do tipo $\mathrm{Cfb}$, segundo classificação climática de Köeppen.

O solo, cujas análises química e física encontram-se na Tabela 1, pertence a classe Latossolo Bruno Alumínico (EMBRAPA, 2006), textura muito argilosa e relevo suave ondulado. Segundo 
CAMARGO et al. (1987), esta classe compreende solos minerais não hidromórficos, muito argilosos, profundos, porosos, acentuadamente drenados, de alta saturação com alumínio trocável e muito ácidos.

O talhão utilizado para instalação do experimento foi implantado a pleno sol, no ano de 1988, empregando o espaçamento $3 \mathrm{~m} \times 2 \mathrm{~m}$ (entrelinhas, entre plantas). Durante a determinação das unidades amostrais, o erval encontrava-se em plena fase produtiva.

TABELA 1 - Resultado da análise do solo realizada para os três blocos da área experimental, na região de Guarapuava-PR.

\begin{tabular}{|c|c|c|c|c|c|c|c|c|c|c|c|c|c|c|c|}
\hline \multirow[b]{2}{*}{ Blocos } & \multicolumn{2}{|c|}{--- pH --- } & $\mathrm{Al}^{+3}$ & $\mathrm{H}^{+}+\mathrm{Al}^{+3}$ & $\mathrm{Ca}^{+2}$ & $\mathrm{Mg}^{+2}$ & $\mathrm{~K}^{+}$ & SB & $\mathrm{T}$ & \multirow{2}{*}{$\begin{array}{c}\mathrm{P} \\
\mathrm{mg} \mathrm{dm}^{-3}\end{array}$} & \multirow{2}{*}{$\begin{array}{c}\mathrm{C} \\
\mathrm{g} \mathrm{dm}^{-3}\end{array}$} & V & M & \multirow{2}{*}{$\begin{array}{c}\mathrm{Ca} / \mathrm{Mg} \\
---\mathrm{g} \mathrm{k}\end{array}$} & \multirow[t]{2}{*}{ Argila } \\
\hline & $\mathrm{CaCl}_{2}$ & SMP & $\ldots$ & & cr & $10 l_{c} d m$ & & & -- & & & ---- & |0----- & & \\
\hline I & 3,60 & 4,50 & 4,30 & 15,20 & 0,13 & 0,08 & 0,09 & 0,30 & 15,50 & 1,90 & 32,90 & 1,94 & 91,68 & 1,6 & 680,0 \\
\hline II & 3,70 & 4,50 & 4,40 & 15,20 & 0,13 & 0,08 & 0,10 & 0,31 & 15,51 & 1,60 & 34,10 & 2,00 & 91,48 & 1,6 & 640,0 \\
\hline III & 3,60 & 4,60 & 4,00 & 14,10 & 0,15 & 0,08 & 0,19 & 0,42 & 14,52 & 1,60 & 32,90 & 2,89 & 86,77 & 1,6 & 560,0 \\
\hline Média & 3,60 & 4,50 & 4,20 & 14,80 & 0,14 & 0,08 & 0,13 & 0,34 & 15,18 & 1,70 & 33,30 & 2,28 & 89,98 & 1,6 & 627,0 \\
\hline
\end{tabular}

Na Figura 1a é possível observar que: (i) o experimento foi conduzido em delineamento blocos ao acaso, perfazendo um total de 3 blocos com 5 tratamentos; (ii) os tipos de poda foram sorteados dentro de cada bloco. Na Figura 1b é possível observar que: (i) cada tratamento foi constituído por 12 plantas podadas, totalizando 72 plantas amostradas em cada bloco; (ii) cada bloco possuía 6 metros de largura por 12 de comprimento, compreendendo uma área de $72 \mathrm{~m}^{2}$; (iii) foi deixada uma fileira de árvores de erva-mate como bordadura.

A coleta dos dados foi realizada entre os dias 13 e 16 de agosto, e consistiu na realização de podas nas plantas de erva-mate, empregando-se cinco tipos de cortes. Na Tabela 2 encontram-se a descrição dos tratamentos utilizados, bem como os procedimentos de coleta dos dados.

\begin{tabular}{|c|c|c|c|c|c|}
\hline Bloco I-T 2 & Bloco I-T 0 & Bloco I-T 4 & Bloco I-T 5 & Bloco I-T, & Bloco I-T \\
\hline Bloco II-T 3 & Bloco $\|-T_{5}$ & Bloco II-T, & Bloco II-T 0 & Bloco $\|-T_{4}$ & Bloco II-T, \\
\hline Bloco III-T, & Bloco III-T 4 & Bloco III-T 2 & Bloco III-T 3 & Bloco III-T 0 & Bloco III-T \\
\hline
\end{tabular}

(a) Blocos e tratamentos do delineamento experimental

\begin{tabular}{|c|c|c|c|c|c|c|c|c|c|}
\hline+ & + & + & + & + & + & + & + & + & + \\
\hline+ & + & $\bullet$ & $\bullet$ & $\bullet$ & $\bullet$ & $\bullet$ & $\bullet$ & + & + \\
\hline+ & + & $\bullet$ & $\bullet$ & $\bullet$ & $\bullet$ & $\bullet$ & $\bullet$ & + & + \\
\hline+ & + & + & + & + & + & + & + & + & + \\
\hline
\end{tabular}

(b) Parcela experimental de cada bloco e tratamento, contendo plantas ( $\bullet$ ) dentro dos blocos, e área de bordadura (+)

FIGURA 1 - "Croquis" da área experimental situada na Fazenda São José, Serra da Esperança, município de Guarapuava-PR, contendo: (a) 3 blocos e 5 tratamentos; (b) parcela contendo a representação das 12 plantas de llex paraguariensis colhidas dentro de cada bloco e tratamento. 
SOUZA, J.L.M. et al. Exportação de nutrientes foliares...

TABELA 2 - Descrição dos tratamentos aplicados em plantas de erva-mate (llex paraguariensis), empregando cinco tipos de poda em diferentes intensidades, na região de Guarapuava-PR.

\begin{tabular}{|c|c|c|}
\hline Tratamento & Tipo de poda & Especificação \\
\hline$T_{0}$ & Sem poda & Tratamento testemunha, onde não foi realizada a poda; \\
\hline $\mathrm{T}_{1}$ & $\begin{array}{l}\text { Poda Toalete } \\
\text { (limpeza) }\end{array}$ & $\begin{array}{l}\text { No tratamento poda de limpeza (Toalete ou suave), poucos ramos } \\
\text { foram removidos, entre } 10 \% \text { e } 20 \% \text { do volume total, procurando } \\
\text { melhorar a condução das plantas; }\end{array}$ \\
\hline $\mathrm{T}_{2}$ & Poda $70 \%$ & $\begin{array}{l}\text { Esta é considerada a poda tradicional, na qual é removida } \\
\text { aproximadamente } 50 \% \text { a } 70 \% \text { da planta; }\end{array}$ \\
\hline $\mathrm{T}_{3}$ & $\begin{array}{l}\text { Recepa } \\
\text { (Rebaixamento) }\end{array}$ & $\begin{array}{l}\text { E uma poda drástica, com a remoção de todas as folhas e ramos da } \\
\text { planta; }\end{array}$ \\
\hline $\mathrm{T}_{4}$ & Desfolha $90 \%$ & Nesta poda apenas $10 \%$ das folhas são deixadas; \\
\hline $\mathrm{T}_{5}$ & $\begin{array}{l}\text { Poda Schier } \\
\text { (comercial) }\end{array}$ & $\begin{array}{l}\text { É realizada em duas etapas. A primeira é realizada na colheita, onde } \\
\text { é feita a desfolha deixando apenas } 10 \% \text { das folhas. A segunda é } \\
\text { realizada no final do inverno, fazendo-se a remoção dos galhos. }\end{array}$ \\
\hline
\end{tabular}

A estimativa da quantidade de nutriente exportada pela cultura da erva-mate foi obtida utilizando a Equação 1:

$$
Q N_{n t}=\frac{\sum_{i=1}^{3} R_{p s / y v} \cdot R m v_{i} \cdot R n_{n i} \cdot M v_{t}}{1.000}
$$

sendo, $Q N_{n t}$ a quantidade do $n$-ésimo nutriente exportado no $t$-ésimo tratamento $\left(\mathrm{kg} \mathrm{ha}^{-1}\right) ; R_{p s / p v}$ é a relação entre a massa seca e fresca das folhas (adimensional); $R m v_{i}$ a relação entre a massa seca e fresca das folhas existentes nas plantas em função dos terços (adimensional); $R n_{n i}$ a relação da quantidade do $n$-ésimo nutriente existente por quilo de folhas de erva-mate no $i$-ésimo terço considerado $\left(\mathrm{g} \mathrm{kg}^{-1}\right)$; $M v_{t}$ a massa fresca de folhas de erva-mate em cada $t$-ésimo tratamento - tipo de poda $(\mathrm{kg}$ $\mathrm{ha}^{-1}$ ); $i$ os terços considerados (inferior, médio e superior); $n$ os nutrientes analisados (N, P, K, Ca e $\mathrm{Mg}$ ); $t$ são os tratamentos que correspondem aos tipos de poda $\left(\mathrm{T}_{1}\right.$ - Toalete ou limpeza, $\mathrm{T}_{2}$ - Poda $70 \%, \mathrm{~T}_{3}$ - Recepa ou rebaixamento, $\mathrm{T}_{4}$ - Desfolha $90 \%$ e $\mathrm{T}_{5}$ - Poda Schier ou comercial). E importante observar que na realização das podas procurou-se retirar uma quantidade de folhas que fosse proporcional a existente em cada terço da planta (inferior, médio e superior).

Para quantificar o $n$-ésimo nutriente exportado no $t$-ésimo tratamento com a Equação 1, a massa fresca das folhas de erva-mate $\left(M v_{t}\right)$ foi determinada no presente experimento considerando cada tipo de poda. As demais relações necessárias foram obtidas a partir de um experimento preliminar (WOLF, 2005), realizado na mesma área experimental, em que os valores encontram-se resumidos e apresentados na Tabela 3 .

TABELA 3 - Resumo das principais relações necessárias para quantificar os nutrientes exportados em função do tipo de poda de acordo com a Equação 1 (WOLF, 2005), em plantas de Ilex paraguariensis, na região de Guarapuava-PR.

\begin{tabular}{|c|c|c|c|c|c|c|}
\hline \multirow{3}{*}{ Terço } & \multicolumn{5}{|c|}{$R n_{n i}$} & \multirow[t]{2}{*}{$R m v$} \\
\hline & $\mathrm{N}$ & $P$ & $\mathrm{~K}$ & $\mathrm{Ca}$ & $\mathrm{Mg}$ & \\
\hline & \multicolumn{5}{|c|}{$\mathrm{g} \mathrm{kg}^{-1}$} & $\%$ \\
\hline Inferior & 17,21 & 0,76 & 14,64 & 5,23 & 3,50 & 35,45 \\
\hline Médio & 20,03 & 0,77 & 15,03 & 4,57 & 2,96 & 36,84 \\
\hline Superior & 19,21 & 0,93 & 15,48 & 3,89 & 2,59 & 27,71 \\
\hline Média Geral & 18,82 & 0,82 & 15,05 & 4,56 & 3,02 & 33,33 \\
\hline
\end{tabular}

$R n_{n i}$ - quantidade de nutriente existente por quilo de folhas de erva-mate nos terços.

$R m v_{i}$ - relação entre a massa seca e fresca da erva-mate extraída das plantas em função dos terços.

$R_{p s / p v}$ - relação da massa seca e fresca das folhas de erva-mate: 0,4463.

No momento da aplicação dos tratamentos, as amostras de solo foram coletadas na projeção da copa das plantas de erva-mate. O solo foi coletado na profundidade entre 0 e $20 \mathrm{~cm}$, sendo tomadas amostras simples, que posteriormente foram homogeneizadas para constituir uma amostra composta para cada bloco. Todas as análises de solo foram realizadas segundo metodologias descritas por EMBRAPA (1997), no Laboratório de Química do Solo, situado no Departamento de Solos e Engenharia Agrícola/ SCA/ UFPR, e os resultados encontram-se na Tabela 1.
Baseando-se nos resultados da análise do solo (Tabela 1), os cálculos para proceder à recomendação da adubação de reposição nitrogenada, fosfatada e potássica, foram realizadas conforme os passos a seguir:

- Cálculo da quantidade de matéria orgânica, a partir da quantidade de C obtida na análise do solo, aplicando a relação de WalkleyBlack e as recomendações de TOMÉ JúNIOR (1997).

- Determinação das recomendações de adubação nitrogenada para a erva-mate (plantio e 
crescimento) e dos teores de matéria orgânica no solo, aplicando-se a Equação 1 e considerando os parâmetros da COMISSÃO DE QUÍMICA E FERTILIDADE DO SOLO -RS/SC (2004);

- Determinação da classe de solo (Classes 1 a 6), faixa do teor de $P$ existente no solo e das recomendações de adubação fosfatada para a ervamate (plantio e reposição), a partir da quantidade de argila obtida na análise do solo $\left(\mathrm{g} \mathrm{kg}^{-1}\right)$ e das recomendações especificadas pela COMISSÃO DE QUÍMICA E FERTILIDADE DO SOLO -RS/SC (2004);

- Determinação dos teores do nutriente, identificação da faixa do teor de $\mathrm{K}$ existente no solo e recomendações de adubação potássica para a erva-mate (plantio e reposição), considerando as recomendações da COMISSÃO DE QUÍMICA E FERTILIDADE DO SOLO -RS/SC (2004);

- Relação entre os teores de nutrientes (N, $\mathrm{P}, \mathrm{K}$ ) exportados pelas folhas de erva-mate e os teores destes mesmos nutrientes determinados em função da análise do solo.

Os dados obtidos para a massa fresca e seca foram submetidos à análise de variância
(ANOVA) $(p<0,05)$ e em seguida, ao teste de comparação de médias por Duncan $(p<0,01)$. Uma planilha eletrônica foi utilizada para armazenar e organizar os dados, e as análises estatísticas foram realizadas utilizando-se o programa "Sirichai's Statistcs" versão 3.0 (1987).

\section{RESULTADOS E DISCUSSÃO}

$\mathrm{Na}$ Tabela 4 estão apresentadas a massa fresca das folhas e galhos (porções do caule sem as folhas) das plantas de erva-mate, considerando os diferentes tipos de poda e blocos do experimento. Como era de se esperar, os tratamentos caracterizados pela Poda $70 \%\left(T_{2}\right)$ e Recepa $\left(T_{3}\right)$ tenderam a apresentar maiores valores médios para a massa de folhas e para a massa de galhos, em relação com as demais podas efetuadas (Tabela 4). Comportamento semelhante também foi observado para a estimativa da massa fresca média das folhas $\left(M v_{t}\right)$, onde os tratamentos $T_{2}(8.504,63$ $\left.\mathrm{kg} \mathrm{ha}^{-1}\right)$ e $\mathrm{T}_{3}\left(9.436,11 \mathrm{~kg} \mathrm{ha}^{-1}\right)$ apresentaram os maiores valores para essa característica (Tabela 5).

TABELA 4 - Massa fresca das folhas e galhos (porções do caule sem as folhas) das plantas de Ilex paraguariensis, em função dos diferentes tipos de poda e blocos do experimento, na região de Guarapuava-PR ( $T_{1}$ - Toalete, $T_{2}$ - Poda $70 \%, T_{3}$ - Recepa, $T_{4}$ - Desfolha, $T_{5}$ - Schier).

\begin{tabular}{ccccccc}
\hline \multirow{2}{*}{ Tratamento } & \multicolumn{3}{c}{ Massa de Folhas } & \multicolumn{2}{c}{ Massa de Galhos } \\
\cline { 2 - 6 } & \multicolumn{1}{c}{ Bloco I } & Bloco II & Bloco III & Bloco I & Bloco II & Bloco III \\
\cline { 2 - 6 } & 21,40 & 22,60 & 17,10 & 7,4 & 6,7 & 6,7 \\
$\mathrm{~T}_{1}$ & 60,50 & 55,80 & 67,40 & 43 & 36,4 & 32,8 \\
$\mathrm{~T}_{2}$ & 80,82 & 69,00 & 54,00 & 109,1 & 92,4 & 69,06 \\
$\mathrm{~T}_{3}$ & 33,35 & 39,50 & 37,70 & - & - & $-1,3$ \\
$\mathrm{~T}_{4}$ & 50,36 & 47,10 & 42,00 & 13,8 & 6,2 & 8,3 \\
$\mathrm{~T}_{5}$ &
\end{tabular}

$\mathrm{T}_{0}$ - todas as plantas não tiveram as folhas e galhos coletados - massa zero.

TABELA 5 - Massa fresca média das folhas de llex paraguariensis $\left(M v_{t}\right)$ em função dos tratamentos testados, na região de Guarapuava-PR ( $\mathrm{T}_{1}$ - Toalete, $\mathrm{T}_{2}$ - Poda 70\%, $\mathrm{T}_{3}$ - Recepa, $\mathrm{T}_{4}$ - Desfolha, $\mathrm{T}_{5}$ - Schier).

\begin{tabular}{crc}
\hline Tratamento & \multicolumn{2}{c}{$M v_{t}$} \\
\cline { 2 - 3 } & ${\mathrm{kg} \mathrm{em} 72 \mathrm{~m}^{2}{ }^{\star *}}^{-1}$ & $\mathrm{~kg} \mathrm{ha}^{-1}$ \\
\hline $\mathrm{T}_{1}$ & $20,37 \mathrm{c}$ & $2.828,70$ \\
$\mathrm{~T}_{2}$ & $61,23 \mathrm{a}$ & $8.504,63$ \\
$\mathrm{~T}_{3}$ & $67,94 \mathrm{a}$ & $9.436,11$ \\
$\mathrm{~T}_{4}$ & $36,85 \mathrm{~b}$ & $5.118,06$ \\
$\mathrm{~T}_{5}$ & $46,49 \mathrm{~b}$ & $6.456,48$ \\
\hline Média & 46,58 & $6.468,80$ \\
\hline
\end{tabular}

$\mathrm{T}_{0}$ - todas as plantas não tiveram as folhas e galhos coletados - massa zero. ${ }^{\star \star}$ Médias seguidas pela mesma letra na coluna não diferem entre si pelo teste de Duncan a $1 \%$ de significância

Os valores do Teste $F$, encontrados na análise de variância revelaram efeitos significativos para os tratamentos de poda aplicados. Nas análises realizadas, obteve-se média igual a $6.468,80 \mathrm{~kg} \mathrm{ha}^{-1}$ quanto às características da massa fresca de folhas, apresentando coeficiente de variação (CV) igual a $12,61 \%(p<0,01)$. Com isso, pode-se afirmar que pelo menos um dos tratamentos (tipo de poda) utilizados no experimento apresenta diferença significativa entre os valores médios de produção de erva-mate.
No entanto, não existiu diferença significativa $(p<0,05)$ entre as médias dos três blocos quanto aos tratamentos testados (tipos de poda), o que indica comportamento semelhante das características mensuradas entre os blocos experimentais.

Em relação à massa fresca média das folhas (Tabela 5) pode-se observar que a poda do tipo Recepa não diferiu significativamente $(p<0,01)$ da Poda $70 \%$, apresentando os maiores valores médios para essa característica analisada. Estes dois tipos de poda representam a maior retirada de 
folhas e galhos de erva-mate em relação aos demais tratamentos testados.

A Poda Schier não diferiu estatisticamente $(p<0,01)$ da poda Desfolha $90 \%$. Como era de se esperar, pela própria característica, a poda do tipo Toalete apresenta a menor retirada de material da área em comparação as demais podas testadas para a cultura de erva-mate (Tabela 5).
A Tabela 6 apresenta, em função dos terços da planta (inferior, médio e superior) e dos diferentes tipos de poda, a quantidade de massa seca e, respectivamente, a quantidade de nutriente que foi exportada. Para possibilitar uma comparação, a Tabela 7 traz as exportações de nutrientes na unidade de $\mathrm{kg} \mathrm{ha}^{-1} \mathrm{ano}^{-1}$ para diferentes sistemas de cultivo realizados por REISSMANN et al. (1985) e CAMPOS (1991).

TABELA 6 - Quantidade de nutriente (N, P, K, CA e Mg) exportado pela cultura de erva-mate, em função dos terços (inferior, médio e superior) e dos diferentes tipos poda $\left(T_{1}-\right.$ Toalete, $T_{2}-$ Poda $70 \%, T_{3}$ Recepa, $\mathrm{T}_{4}$ - Desfolha, $\mathrm{T}_{5}$ - Schier).

\begin{tabular}{|c|c|c|c|c|c|c|}
\hline \multirow{3}{*}{ Nutrientes } & \multirow{3}{*}{ Terços } & \multicolumn{5}{|c|}{ Exportação de nutriente nos tratamentos } \\
\hline & & \multicolumn{5}{|c|}{$\mathrm{kg} \mathrm{ha}^{-1} \mathrm{ano}^{-1}$} \\
\hline & & $\mathrm{T}_{1}$ & $\mathrm{~T}_{2}$ & $\mathrm{~T}_{3}$ & $\mathrm{~T}_{4}$ & $\mathrm{~T}_{5}$ \\
\hline \multirow{4}{*}{ Nitrogênio } & Inferior & 7,70 & 23,16 & 25,69 & 13,94 & 17,58 \\
\hline & Médio & 9,32 & 28,01 & 31,08 & 16,86 & 21,26 \\
\hline & Superior & 6,72 & 20,20 & 22,42 & 12,16 & 15,34 \\
\hline & Total & 23,74 & 71,37 & 79,19 & 42,95 & 54,18 \\
\hline \multirow{4}{*}{ Fósforo } & Inferior & 0,34 & 1,02 & 1,13 & 0,62 & 0,78 \\
\hline & Médio & 0,36 & 1,08 & 1,19 & 0,65 & 0,82 \\
\hline & Superior & 0,33 & 0,98 & 1,09 & 0,59 & 0,74 \\
\hline & Total & 1,02 & 3,08 & 3,41 & 1,85 & 2,34 \\
\hline \multirow{4}{*}{ Potássio } & Inferior & 6,55 & 19,70 & 21,86 & 11,85 & 14,95 \\
\hline & Médio & 6,99 & 21,02 & 23,32 & 12,65 & 15,96 \\
\hline & Superior & 5,42 & 16,28 & 18,06 & 9,80 & 12,36 \\
\hline & Total & 18,96 & 57,00 & 63,24 & 34,30 & 43,27 \\
\hline \multirow{4}{*}{ Cálcio } & Inferior & 2,34 & 7,04 & 7,81 & 4,23 & 5,34 \\
\hline & Médio & 2,13 & 6,39 & 7,09 & 3,85 & 4,85 \\
\hline & Superior & 1,36 & 4,09 & 4,54 & 2,46 & 3,11 \\
\hline & Total & 5,83 & 17,52 & 19,44 & 10,54 & 13,30 \\
\hline \multirow{4}{*}{ Magnésio } & Inferior & 1,57 & 4,71 & 5,23 & 2,83 & 3,58 \\
\hline & Médio & 1,38 & 4,14 & 4,59 & 2,49 & 3,14 \\
\hline & Superior & 0,91 & 2,72 & 3,02 & 1,64 & 2,07 \\
\hline & Total & 3,85 & 11,57 & 12,84 & 6,96 & 8,79 \\
\hline
\end{tabular}

$\mathrm{T}_{0}$ - todas as plantas não tiveram as folhas e galhos coletados - massa zero.

TABELA 7 - Exportações de nutrientes para a cultura de llex paraguariensis verificada em um ano para diferentes sistemas de cultivo.

\begin{tabular}{lcccccc}
\hline \multirow{2}{*}{ Sistema de Cultivo } & \multicolumn{5}{c}{ Exportação de Nutrientes } \\
\cline { 2 - 6 } & \multicolumn{5}{c}{$\mathrm{kg} \mathrm{ha}^{-1} \mathrm{ano}^{-1}$} \\
\cline { 2 - 6 } & $\mathrm{N}$ & $\mathrm{P}$ & $\mathrm{K}$ & $\mathrm{Ca}$ & $\mathrm{Mg}$ \\
\hline${ }^{*} 700$ árvores por ha, safra & 75,0 & 3,6 & 63,0 & 30,0 & 18,0 \\
${ }^{* *} 12$ anos, safra & 93,5 & 4,5 & 46,8 & 28,2 & 23,2 \\
${ }^{* *} 12$ anos, safrinha & 150,6 & 7,1 & 92,0 & 39,0 & 34,2 \\
\hline
\end{tabular}

Fontes: * REISSMANN et al., 1985; ${ }^{* *}$ CAMPOS (1991)

Com exceção do $\mathrm{Ca}$ e $\mathrm{Mg}$, os valores de exportação de nutrientes $\mathrm{N}, \mathrm{P}, \mathrm{K}$ apresentados por REISSMANN et al. (1985) (Tabela 7) foram muito próximos aos encontrados no presente trabalho para o tratamento Recepa. Comportamento semelhante foi observado para a Poda $70 \%$, principalmente em relação ao $\mathrm{N}$ e $\mathrm{P}$ (Tabela 6 ). Os resultados apresentados por REISSMANN et al. (1985) permitem comparar os valores obtidos no presente trabalho e supor que a quantidade de biomassa retirada em seu experimento foi similar aos tratamentos Poda 70\% $\left(\mathrm{T}_{2}\right)$ e Recepa $\left(\mathrm{T}_{3}\right)$. 
CAMPOS (1991) empregou uma poda denominada comercial em um erval com 12 anos de idade, durante a safra e safrinha. Em relação aos dados apresentados por esse autor (Tabela 7), pode-se verificar que durante a safra, com exceção do $\mathrm{K}$, todos os nutrientes apresentaram valores superiores aos tratamentos de poda $\left(T_{1}, T_{2}, T_{3}, T_{4} e\right.$ $\mathrm{T}_{5}$ ) testados no presente estudo. Todavia, é importante destacar a similaridade dos teores dos nutrientes $\mathrm{N}, \mathrm{P}$ e Ca em relação aos obtidos no $T_{3}$ (Tabela 6). Esse efeito não ocorreu durante a safrinha, onde todos os teores de nutrientes apresentaram, aproximadamente, o dobro dos valores encontrados nos tratamentos de poda utilizados (Tabela 6).

Existe uma série de fatores que podem ter influenciado esse comportamento diferenciado. A idade das folhas afeta a distribuição de nutrientes em função da redistribuição dos nutrientes móveis, como o N, P, K, Mg, para outros órgãos (VAN DEN DRIESSCHE, 1984; EPSTEIN e BLOOM, 2004). Além disso, alguns trabalhos relatam que os teores de nutrientes são específicos não somente para a espécie, como também, dependem da sanidade, época, juvenilidade do material, idade, ambiente e fatores genéticos e ontogenéticos (FOSSATI, 1997; REISSMANN et al., 1997; RACHWAL et al., 2000; PANDOLFO et al., 2003; BORILLE, 2004; RAKOCEVICZ et al., 2006; OLIVA, 2007).
É interessante observar que a Poda $70 \%$ e Recepa são os tratamentos que apresentam a melhor concordância e se enquadram de forma mais adequada dentro dos teores de nutrientes exportados, verificados por outros autores que trabalharam com erva-mate.

A interpretação dos resultados da análise do solo aliada as recomendações da COMISSÃO DE FERTILIDADE DO SOLO-RS/SC (2004), permitiram verificar que (Tabela 8): (i) o teor de matéria orgânica no solo encontrado nos blocos I, II e III classifica-se com alto, sendo necessário a aplicação de até $20 \mathrm{~kg} \mathrm{~N} \mathrm{ha}^{-1}$ na realização do plantio e até $60 \mathrm{~kg} \mathrm{~N} \mathrm{ha}^{-1}$ na fase de crescimento da planta; (ii) o solo dos três blocos é identificado como Classe 1 (teor de argila $>55 \%$ ) e possui "faixa de teor de $\mathrm{P}$ no solo" muito baixo, sendo necessário a aplicação de $90 \mathrm{~kg} \mathrm{P}_{2} \mathrm{O}_{5} \mathrm{ha}^{-1}$ no plantio e $120 \mathrm{~kg} \mathrm{P}_{2} \mathrm{O}_{5} \mathrm{ha}^{-1}$ na reposição; (iii) a faixa do teor de $\mathrm{K}$ foi identificada com muito baixa para os solos dos blocos I e II e médio para o bloco III, sendo recomendado $40 \mathrm{~kg}$ $\mathrm{K}_{2} \mathrm{O}$ ha $^{-1}$ para o plantio e $60 \mathrm{~kg} \mathrm{~K}_{2} \mathrm{O}$ ha $^{-1}$ para a reposição; (iv) o valor médio de $\mathrm{K}$ encontrado no solo amostrado corresponde a $0,38 \mathrm{cmol}_{\mathrm{cm}}^{-3}$, e encontra-se dentro dos limites classificados pela EMATER (1991), ou seja, valores entre 0,12 e 0,46 cmol $\mathrm{kg}^{-1}$ (ou $0,046 \mathrm{~g} \mathrm{~kg}^{-1}$ e $0,179 \mathrm{~g} \mathrm{~kg}^{-1}$ ). FOSSATI (1997) e REISSMANN et al. (1983) encontraram teores de $\mathrm{K}$ entre $0,042 \mathrm{~g} \mathrm{~kg}^{-1}$ e $0,093 \mathrm{~g} \mathrm{~kg}^{-1}$.

TABELA 8 - Teores de N, P, K exportados pelas folhas de llex paraguariensis e teores destes mesmos nutrientes obtidos a partir da análise do solo onde se encontra o experimento $\left(T_{1}\right.$ - Toalete, $T_{2}-$ Poda $70 \%, T_{3}$ - Recepa, $\mathrm{T}_{4}$ - Desfolha, $\mathrm{T}_{5}$ - Schier).

\begin{tabular}{cccccccc}
\hline \multirow{3}{*}{ Nutriente } & \multicolumn{7}{c}{ Recomendação de adubação } \\
\cline { 2 - 7 } & Plantio $^{(1)}$ & Reposição $^{(1)}$ & $\mathrm{T}_{1}{ }^{(2)}$ & $\mathrm{T}_{2}{ }^{(2)}$ & $\mathrm{T}_{3}{ }^{(2)}$ & $\mathrm{T}_{4}{ }^{(2)}$ & $\mathrm{T}_{5}{ }^{(2)}$ \\
\hline $\mathrm{N}$ & 20 & 60 & 23,74 & 71,37 & 79,19 & 42,95 & 54,18 \\
$\mathrm{P}_{2} \mathrm{O}_{5}{ }^{*}$ & 90 & 120 & 1,02 & 3,08 & 3,41 & 1,85 & 2,34 \\
$\mathrm{~K}_{2} \mathrm{O}^{* *}$ & 40 & 60 & 18,96 & 57,00 & 63,24 & 34,30 & 43,27 \\
\hline
\end{tabular}

(1) COMISSÃO DE FERTILIDADE DO SOLO-RS/SC (2004).

(2) Estimativa na exportação de nutrientes em diferentes tipos de poda na planta de erva-mate.

A Tabela 8 apresenta os teores de N, P e K exportados nas folhas das plantas de erva-mate e os teores destes mesmos nutrientes obtidos a partir da análise do solo onde se encontram as plantas do experimento. Essa tabela permite comparar a quantidade de nutriente disponível no solo e o quanto a planta exporta para as folhas, visando estabelecer a reposição necessária para manter a sustentabilidade da planta.

A COMISSÃO DE FERTILIDADE DO SOLORS/SC (2004) recomenda a reposição no solo de $60 \mathrm{~kg} \times \mathrm{ha}^{-1}$ de $\mathrm{N}$. Esse valor foi superior a exportação de nutriente verificada na Poda Schier (T5 - 54,18 kg $\mathrm{ha}^{-1}$ ), Desfolha (T5 - 42,95 $\mathrm{kg} \mathrm{ha}^{-1}$ ) e Toalete (T5 $23,74 \mathrm{~kg} \mathrm{ha}^{-1}$ ), porém inferior $18,95 \%$ ao valor encontrado na Poda $70 \%\left(T_{2}-71,37 \mathrm{~kg} \mathrm{ha}^{-1}\right)$ e $32,0 \%$ na Recepa $\left(79,19 \mathrm{~kg} \mathrm{ha}^{-1}\right)$.

Como a Poda $70 \%$ corresponde ao manejo mais empregado na condução dos ervais da Fazenda São José, Guarapuava-PR, verifica-se que a realização de uma adubação nitrogenada seguindo as recomendações da COMISSÃO DE FERTILIDADE DO SOLO-RS/SC (2004) poderá levar a uma deficiência do referido nutriente à cultura da ervamate. Esta deficiência poderá resultar em uma redução na massa fresca das folhas e galhos (SOSA, 1994; PRAT KRICUN e BELINGHERI, 1995) e limitar a produção de matéria seca (BELLOTE e STURION,1985).

REISSMANN et al. (1983) recomendam que a exportação de nutrientes através da colheita deve ser compensada mediante a quantificação da biomassa exportada e da determinação do conteúdo de nutrientes nela existente, visando manter a capacidade produtiva da cultura.

Os valores de adubação de reposição do $K$ recomendados pela COMISSÃO DE FERTILIDADE DO SOLO-RS/SC (2004) para a cultura da erva-mate na área experimental $\left(60 \mathrm{~kg} \mathrm{ha}^{-1}\right)$ ficou muito próximo do que as Podas $70 \%\left(57,0 \mathrm{~kg} \mathrm{ha}^{-1}\right)$ e Recepa $(63,24$ $\mathrm{kg} \mathrm{ha}^{-1}$ ) exportam para as folhas, apresentando um desvio positivo de $5,0 \%$ e negativo em $5,4 \%$, 
respectivamente. Esse resultado é bom, pois a Poda $70 \%$ é a mais utilizada na propriedade São José e toda a região de Guarapuava-PR. No entanto, é importante observar que a recomendação da COMISSÃO DE FERTILIDADE DO SOLO-RS/SC (2004) para o K apresenta um desvio positivo de $68,4 \%, 42,8 \%$ e $27,9 \%$, respectivamente, para o que se exporta para as folhas nas podas Toalete $\left(T_{1}\right)$, Desfolha $\left(T_{4}\right)$ e Poda Schier $\left(T_{5}\right)$.

O P apresentou valores exportados para as folhas da planta de erva-mate entre $1,02 \mathrm{~kg} \mathrm{ha}^{-1} \mathrm{na}$ poda Toalete $\left(T_{1}\right)$ a $3,41 \mathrm{~kg} \mathrm{ha}^{-1}$ na poda Recepa $\left(\mathrm{T}_{3}\right)$. Esses valores são muito menores do que os $120 \mathrm{~kg} \mathrm{ha}^{-1}$ recomendados pela COMISSÃO DE FERTILIDADE DO SOLO-RS/SC (2004) para reposição do referido nutriente na área experimental onde se encontra a cultura (Tabela 8). Não se deve esquecer, porém, que o $\mathrm{P}$ pode se fixar no solo e a quantidade a ser fornecida deverá ser maior do que os valores exportados para as folhas da planta. A quantificação exata depende de testes de calibração envolvendo a produção de biomassa e o estado nutricional em relação ao fornecimento de níveis crescentes de $\mathrm{P}$, bem como do fertilizante utilizado.

No entanto, as recomendações de $P$ para o plantio $\left(90 \mathrm{~kg} \mathrm{ha}^{-1}\right)$ e reposição $\left(120 \mathrm{~kg} \mathrm{ha}^{-1}\right)$ do $P$, encontradas na COMISSÃO DE FERTILIDADE DO SOLO-RS/SC (2004), podem mesmo estar além das necessidades da cultura da erva-mate no local do experimento. Esta afirmativa baseia-se nas seguintes considerações: PANDOLFO et al. (2000) comentam que a aplicação de $P$ na cultura de ervamate não proporciona diferença significativa na sua produção; REISSMANN et al. (1983) e RACHWAL et al. (2000), também obtiveram baixos teores de $P$ nas folhas da erva-mate, evidenciando baixa exigência do elemento pela planta.

\section{CONCLUSÕES}

1) Os teores totais dos nutrientes $(\mathrm{N}, \mathrm{P}, \mathrm{K}$, $\mathrm{Ca}$ e $\mathrm{Mg}$ ) obtidos nas folhas da erva-mate foram menores do que os encontrados na literatura em ervais nativos do Paraná;

2) A poda do tipo Recepa exporta $70,0 \%$, $9,9 \%, 45,8 \%$ e $31,6 \%$ a mais de biomassa e nutriente (N, P, K, Ca e Mg) do que as podas do tipo Toalete, Poda 70\%, Desfolha $90 \%$ e Schier, respectivamente;

3) Os teores de nutrientes exportados $(\mathrm{kg}$ ha $^{-1}$ ) na Poda $70 \%$ e Recepa são os que mais se aproximam dos valores apresentados e discutidos na literatura, indicando que podas intermediárias aos dois tipos são as mais utilizadas nas pesquisas envolvendo poda da erva-mate;

4) A relação entre adubação recomendada a partir da análise do solo e os teores de nutrientes exportados para folhas, indica: (i) coerência para os nutrientes N (Poda Schier) e K (Poda 70\% e Recepa), e incoerência para o nutriente $P$ em todos os tipos de poda analisados; (ii) déficit de $19,0 \%$ na adubação para o $\mathrm{N}$ na poda mais utilizada na região de Guarapuava-PR (Poda 70\%).

\section{REFERÊNCIAS}

1. BELLOTE, A.F.J.; STURION, J.A. Deficiências minerais em erva-mate (Ilex paraguariensis St. Hil.). In: SEMINÁRIO SOBRE ATUALIDADES E PERSPECTIVAS FLORESTAIS, 1985, Curitiba. Anais. Curitiba, 1985. p. 124-127. (Documentos, 15).

2. BISSO, F.P.; SALET, R.L. Exportação de nutrientes pela poda de erva-mate (Ilex paraguariensis St.Hill.). Santa Maria: Departamento de Solos UFSM, 2000. 1 CD-ROM.

3. BORILLE, A.M.W. Relação entre compostos fitoquimicos e o nitrogênio em morfotipos de erva-mate (Ilex paraguariensis St. Hil.). Curitiba, 2004. 109 f. Dissertação (Mestrado em Agronomia) - Setor de Ciências Agrárias, Universidade Federal do Paraná.

4. CAMARGO, M.N.; KLAMT, E.; KAUFFMAN, J.H. Classificação de solos usado em levantamentos pedológicos no Brasil. Boletim Informativo da Sociedade Brasileira do Solo, v.12, n.1, p.11-33, 1987.

5. CAMPOS, M.A.A. Balanço de biomassa e nutrientes em povoamentos de Ilex paraguariensis: avaliação na safra e na safrinha. Curitiba, 1991. $107 \mathrm{f}$. Dissertação (Mestrado em Engenharia Florestal) - Universidade Federal do Paraná.

6. CARPANEZZI, A.A. Banco de sementes e deposição de folhedo e seus nutrientes em povoamentos de bracatinga (Mimosa scabrella Bentham) na Região Metropolitana de Curitiba-PR. Rio Claro, 1997. 170 f. Tese (Doutorado) - Universidade Estadual Paulista Julio de Mesquita Filho.

7. COMISSÃO DE QUÍMICA E FERTILIDADE DO SOLO - RS/SC. Manual de adubação e calagem para os Estados do Rio Grande do Sul e de Santa Catarina. 10. ed. Porto Alegre: Comissão de Química e Fertilidade do Solo - RS/SC, 2004.

8. DA CROCE. D.M.; FLOSS, P.A. Comportamento de procedência de erva-mate (Ilex paraguariensis St.Hil.) para a região oeste e norte de Santa Catarina. Curitiba: EMBRAPA/CNPF, 1993. (Relatório de pesquisa).

9. DA CROCE, D.M.; FLOSS, P.A. Cultura da erva-mate no Estado e Santa Catarina. Florianópolis: EPAGRI, 1999. 81p. (Boletim Técnico, 100).

10. DONADUZZI, C.M.; CARDOZO JÚNIOR, E.L.; DONADUZZI, E.M.; MANFIO, J.L. Avaliação da presença de contaminantes microbiológicos em amostras de erva-mate (Ilex paraguariensis St. Hil.) comercializadas em embalagens de papel e laminados. In: CONGRESSO SUL-AMERICANO DA ERVA-MATE, 3., FEIRA DO AGRONEGÓCIO DA ERVA-MATE, 1., 2003, Chapecó. Anais. Chapecó: EPAGRI, 2003. 1 CD-ROM.

11. EMATER. Empresa Paranaense de Assistência Técnica e Extensão Rural. Manual da erva-mate. 2 ed. Curitiba, 1991. $104 \mathrm{p}$.

12. EMBRAPA. Empresa Brasileira de Pesquisa Agropecuária. Sistema brasileiro de classificação de solos. SANTOS, H. G. [et al.] (Ed.). 2 ed. Rio de Janeiro: Embrapa Solos, 2006. 306 p.

13. EMBRAPA. Empresa Brasileira de Pesquisa Agropecuária. Manual de métodos de análise de solo. 2. ed. Rio de Janeiro: Embrapa Solos, 1997. 
14. EPSTEIN, E.; BLOOM, A. Nutrição mineral de plantas: princípios e perspectivas. Tradução: Maria Edna Tenório Nunes. Ed. 2. Londrina: Editora Planta, 2004. 403 p.

15. FOSSATI, L.C. Avaliação do estado nutricional e da produtividade da erva-mate (Ilex paraguariensis St. Hil.) em função do sítio e da dioicia. Curitiba, 1997. 113 f. Dissertação (Mestrado em Engenharia Florestal) - Universidade Federal do Paraná.

16. GAIAD, S., LOPES, E.S. Ocorrência de micorriza vesicular-arbuscular em erva-mate (Ilex paraguariensis St. Hil.). Boletim de Pesquisa Florestal, v.12, p. 21-29, 1986.

17. GOMES, F.S., PESSOTTI, J.E.S., PACHECO, R.M. Exportação de nutrientes por clones de Eucalyptus urophylla, em três unidades de solo no vale do Rio Jari. In: IUFRO CONFERENCE ON SILVICULTURE AND IMPROVEMENT OF EUCALYPT, 1997, Salvador. Anais., Salvador, 1997. v. 3. p. 209-214

18. LOURENÇO, R.S.; CURCIO, G.R.; RACHWAL, M.G.; MEDRADO, M.J.S. Avaliação de níveis de nitrogênio sobre a produção de erva-mate (Ilex paraguariensis St. Hil.) em Fernandes Pinheiro, PR, em Latossolo Vermelho escuro. Boletim de Pesquisa Florestal, v. 34, p. 75-98, 1997.

19. MARQUES, R.; BENGHI, C.P. Aporte de nutrientes por frações da serrapilheira em sucessão ecológica de um ecossistema da floresta atlântica. Revista Floresta, v. 33, n. 3, p. 257-264, 2003.

20. OLIVA, E.V. Composição química e produtividade de procedências e progênies de erva-mate (IIex paraguariensis St. Hil.) cultivadas em Latossolo Vermelho Distrófico no município de Ivaí - PR. Curitiba, 2007. 73 f. Dissertação (Mestrado em Ciência do Solo) - Universidade Federal do Paraná.

21. PANDOLFO, C.M.; VEIGA, M.; CERETTA, C.A. Plano estadual: Alterações em características químicas do solo com aplicação de fontes de nutrientes, em cinco sistemas de manejo do solo. Santa Maria: EPAGRI/EECN, 2000.

22. PANDOLFO, C.M.; FLOSS, P.A.; DA CROCE, D.M.; DITTRICH, R.C. Resposta da erva-mate (Ilex paraguariensis St. Hil.) à adubação mineral e orgânica em Latossolo Vermelho Aluminoférrico. Ciência Florestal, v. 13, n. 2, p. 37-45. 2003.

23. PARANÁ. Secretaria de Estado da Agricultura e do Abastecimento. Plano estadual da erva-mate: demandas e recursos priorizados a nível dos pólos regionais. Curitiba, 1997.

24. PASINATO, R. Aspectos etnoentomológicos, socioeconômicos e ecológicos relacionados à cultura da erva-mate (Ilex paraguariensis) no município de Salto do Lontra, Paraná, Brasil. São Paulo, 2003. 112 f. Dissertação (Mestrado em Ecologia de Agroecossistemas) - Escola Superior de Agricultura Luiz de Queiroz.

25. PRAT KRICUN, S.D.; BELINGHERI, L.D. Recolección de especies silvestres y cultivadas del género en las provincias de Misiones y Tucumán (Argentina) y en los Estados de Paraná, Santa Catarina y Rio Grande do Sul (Brasil), período 19881992. In: ERVA-MATE: BIOLOGIA E CULTURA NO CONE SUL, 1995, Porto Alegre. Anais. Porto Alegre: Editora da Universidade, UFRGS, 1995. p. 313-321.

26. RACHWAL, M.F.G.; CURCIO, G.R.; DEDECEK, R.A.; NIETSCHE, K.; RADOMSKI, M.I. Influência da luminosidade sobre os teores de macronutrientes e taninos em folha de erva-mate. In: CONGRESSO SUL-AMERICANO DE ERVA-MATE, 2; REUNIÃO TÉCNICA DA ERVA-MATE, 3, 2000, Porto Alegre. Anais. Porto Alegre: Ed. Dos Organizadores, 2000. p. 417420.

27. RAKOCEVICZ, M.; MEDRADO, M.J.S.; LUCAMBIO, F.; VALDUGA, T.A. Influência do Sexo, da Sombra e da Idade da Folhas no Sabor do Chimarrão. In: CONGRESO SUDAMERICANO DE LA YERBA MATE, 4, REUNIÓN TÉCNICA DE LA YERBA MATE, 4, EXPOSICIÓN DE AGRONEGOCIOS DE LA YERBA MATE, 2, Posadas. Anais. Posadas: INYM, 2006. p. 31-36.

28. REISSMANN, C.B.; KOEHLER, C.W.; ROCHA, H.O. da.; HILDEBRAND, E.E. Avaliação das exportações de macronutrientes pela exploração da erva-mate. In: SEMINÁRIO SOBRE ATUALIDADES E PERSPECTIVAS FLORESTAIS, 10: Silvicultura da erva-mate (Ilex paraguariensis St. Hil.), 1985, Curitiba. Anais. Curitiba: EMBRAPA-CNPF, 1985. p. 128-139.

29. REISSMANN, C.B.; PREVEDELLO, B.M.S.; QUADROS, R.M.B de; RADOMSKI, M.I. production and foliar N, P, K, Ca and Mg levels in erva-mate (llex paraguariensis St. Hil.) related to increasing base saturation levels. Brazilian Archives of Biology and Technology, v.40, n.1, p.241- 249, 1997.

30. REISSMANN, C.B.; ROCHA, H.; KOELHLER, C.W.; CALDAS, R.L.S.; HILDEBRAND, E.E. Bioelementos em folhas e hastes de erva-mate (Ilex paraguariensis St. Hil.) sobre Cambissolos na região de Mandirituba-PR. Revista Floresta, v. 14, n. 2, p. 49-54, 1983

31. RODIGHERI, H.R.; PENTEADO JÚNIOR, J.; BUSSATA, L.A.; FERRON, R.M.; MOSELE, S. H. Rentabilidade econômica do consórcio erva-mate e milho na região de Erechim - RS. Colombo: EMBRAPA-CNPF, 1995. 16 p.

32. SOSA, D.A. Fertilizacion quimica: abonos, requerimientos nutricionais. In: CURSO DE CAPACITACIÓN EN PRODUCCIÓN D E YERBA-MATE, 2., 1994, Cerro Azul. Curso. Cerro Azul: INTA - Estacion Experimental Agropecuaria Cerro Azul, 1994. p. 68-85

33. TOMÉ JÚNIOR, B. Manual para interpretação de análise de solo. Guaíba: Agropecuária, 1997. 247p.

34. VAN DEN DRIESSCHE, R. Nutrient storage, retranslocation and relation ship of stress to nutrition. In: NUTRITION OF PLANTATION FOREST, 1984, London. Anais. London: Academic Press/Bowen, 1984. p. 181-209.

35. VETTORI, L. Métodos de análise do solo. Rio de Janeiro: Divisão de Pedologia e Fertilidade do Solo, 1969. 24 p. (Boletim Técnico n. 7)

36. WOLF, C.S. Estimativa da exportação de nutrientes foliares em diferentes tipos de poda na cultura da ervamate (Ilex paraguariensis St. Hil.). Curitiba, 2005. 65 f. Dissertação (Mestrado em Ciência do Solo) - Universidade Federal do Paraná. 
